\title{
Infected Periapical Cemental Dysplasia of the Mandible a Surgical Approach: Case Report
}

\author{
Federico Lamus DDS* \\ University of Missouri, USA
}

Submission: April 06, 2016; Published: August 29, 2016

*Corresponding author: Federico Lamus DDS, University of Missouri-Kansas City, Heartland Dental Group, Leavenworth, KS, USA.

\begin{abstract}
Osseous dysplasias are non-neoplastic benign fibrous-osseous lesions that result from a localized change of normal bone in which all the components of cancellous bone are replaced by fibrous and cementum like tissues containing different amounts of abnormal appearing bone. The solitary (monostotic) type which occurs typically in middle age; the mean age is 39 years and occurs nine times more often in females than males and almost three times more often in blacks than in whites, it's also frequently seen in Asians. Cone Beam Computed Tomography (CBCT) has become a commonly used diagnostic tool in Dentistry when periapical surgery is contemplated to be able to gain knowledge about the relationship of the lesions and their neighboring anatomical features.
\end{abstract}

\section{Case Presentation}

A 66 year old Asian Female presented to our office for her 3 month routine periodontal maintenance appointment, her health history was evaluated and the patient had been diagnosed with high blood pressure and epilepsy, conditions for which she was taking Depakote and Mavik, no history of Biophosphonate intake, heavy bleeding and suppuration was reported by the hygienist during probing at the location of tooth \#19 (distal) (Figure 1).

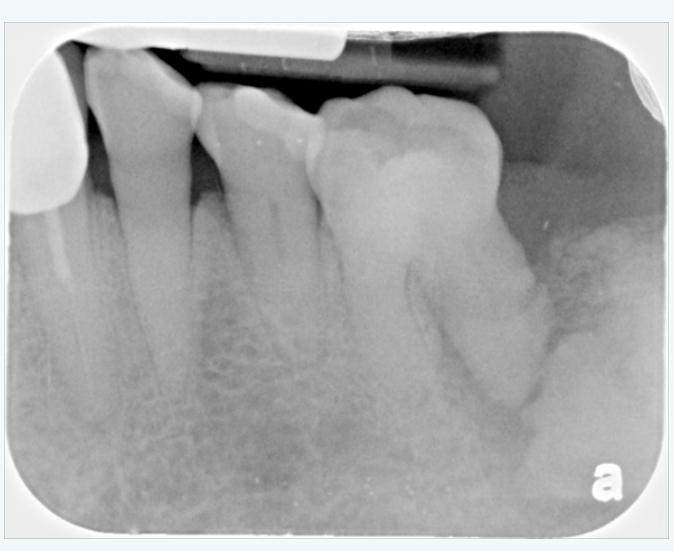

Figure 1: Heavy bleeding and suppuration was reported by the hygienist during probing at the location of tooth \#19 (distal).

A fibrous osseous lesion, diagnosed as Periapical Cemental Dysplasia had been noted on the chart as an incidental finding during the evaluation of a small full mouth survey taken 5 years earlier, everything had been found to be within normal limits and free of symptoms at the time of the initial examination, the Periodontist examined the patient after the hygienist informed her of the finding, upon clinical inspection it was found that tooth \#19 had mobility class lll, deep pocketing (12 mm), profuse bleeding, suppuration and pain upon percussion and pressure tests, the tooth was deemed non-restorable due to the advance state of deterioration in the periodontal tissues and an extraction of the tooth was recommended, she was referred to the Oral Surgeon. The tooth (\#19) was removed by the Oral surgeon under local anesthetic using $72 \mathrm{mg}$ of Lidocaine $2 \%$ using an inferior alveolar block and the tooth was removed without complications.

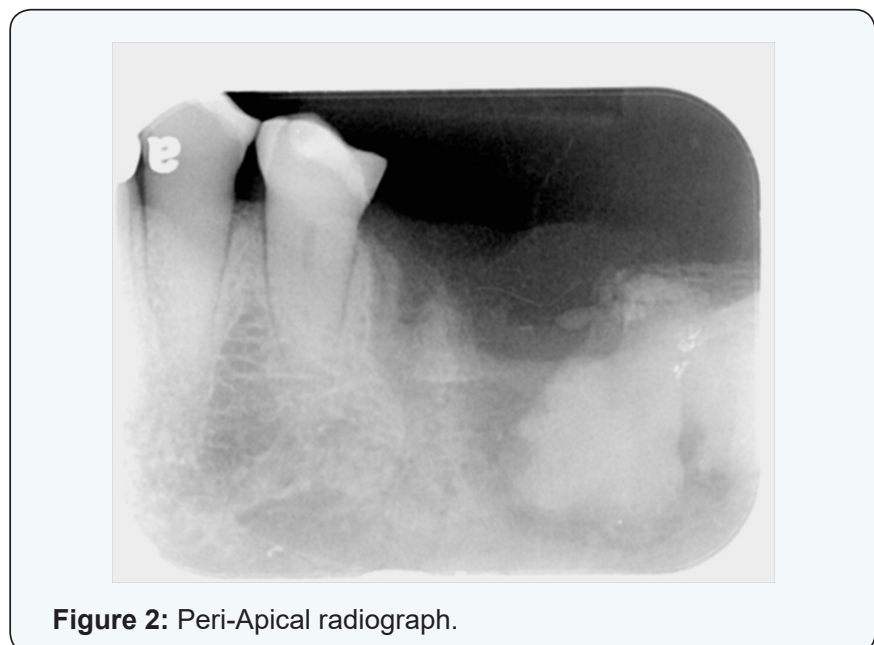

Two months later the patient presented to our office with a chief complaint of purulent drainage coming from the extraction 
site of where tooth \#19 was located at along with mild vestibular swelling and erythema, a Peri-Apical radiograph was taken and revealed increased lucency around the previously noted fibrous osseous lesion, no history of pain, trismus and airway issues were noted. She was placed on $500 \mathrm{mg}$ of Cipro orally every 12 hours for one week and was scheduled for a re-evaluation with the Oral Surgeon one week later. A week later the patient presented with purulent discharge still present but no pain or bleeding was noted at the time (Figure 2). A CBCT scan was taken to evaluate the lesion, size, location and vital structures associated with the it; A limited Volume maxillofacial non-contrast, thin slices $(0.133 \mu \mathrm{m}$ resolution), Cone-Beam CT, $85 \mathrm{kV} 10 \mathrm{~mA}$ for $6 \mathrm{~s}$, (503mGy.cm $\left.{ }^{2}\right)$ of the left body of the mandible was taken.

\section{General Findings}

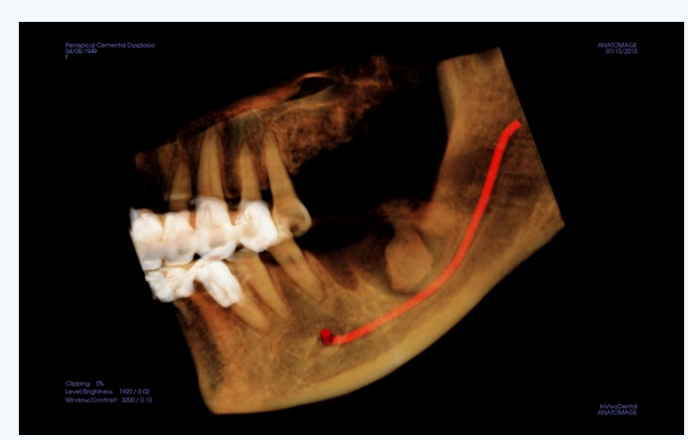

Figure 3: СВСТ scan.

Teeth \# 8, 9,14-19, 23 \& 24 are not present on the volume obtained, there are coronal restoration on teeth \# 10, 11, 12 \&

\section{2 (Figure 3)}

\section{Specific Findings}

On the left body of the mandible there is radiopaque entity (Figure 4,5) surrounded by a radiolucent band at the level of where teeth \# 17-19 were located at, the radiolucent band encapsulates the entire lesion and the width is approximately $2 \mathrm{~mm}$ in its entirety, the radiopaque lesion measures approximately $10.65 \mathrm{~mm}$ (mesial-distal) x $7.62 \mathrm{~mm}$ (buccal-lingual) $\times 14.35$ $\mathrm{mm}$ (coronal-apically), the lesion has similar density as dental root structure, the lingual cortical plate is slightly expanded, the lesion reaches the inferior alveolar canal and the coronal-buccal aspect of the lesion appears to be thinning the cortical plate with evidence of dis-rupture noted.

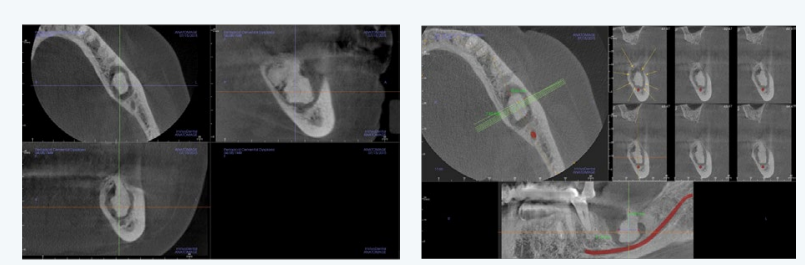

Figures 4 \& 5: On the left body of the mandible there is radiopaque entity.

There is no clinical report of any paresthesia. The radiographic impression was periapical osseous dysplasia with an infected involucrum [1]. The treatment plan presented was for surgical removal and biopsy of the infected involucrum under local anesthesia [2].
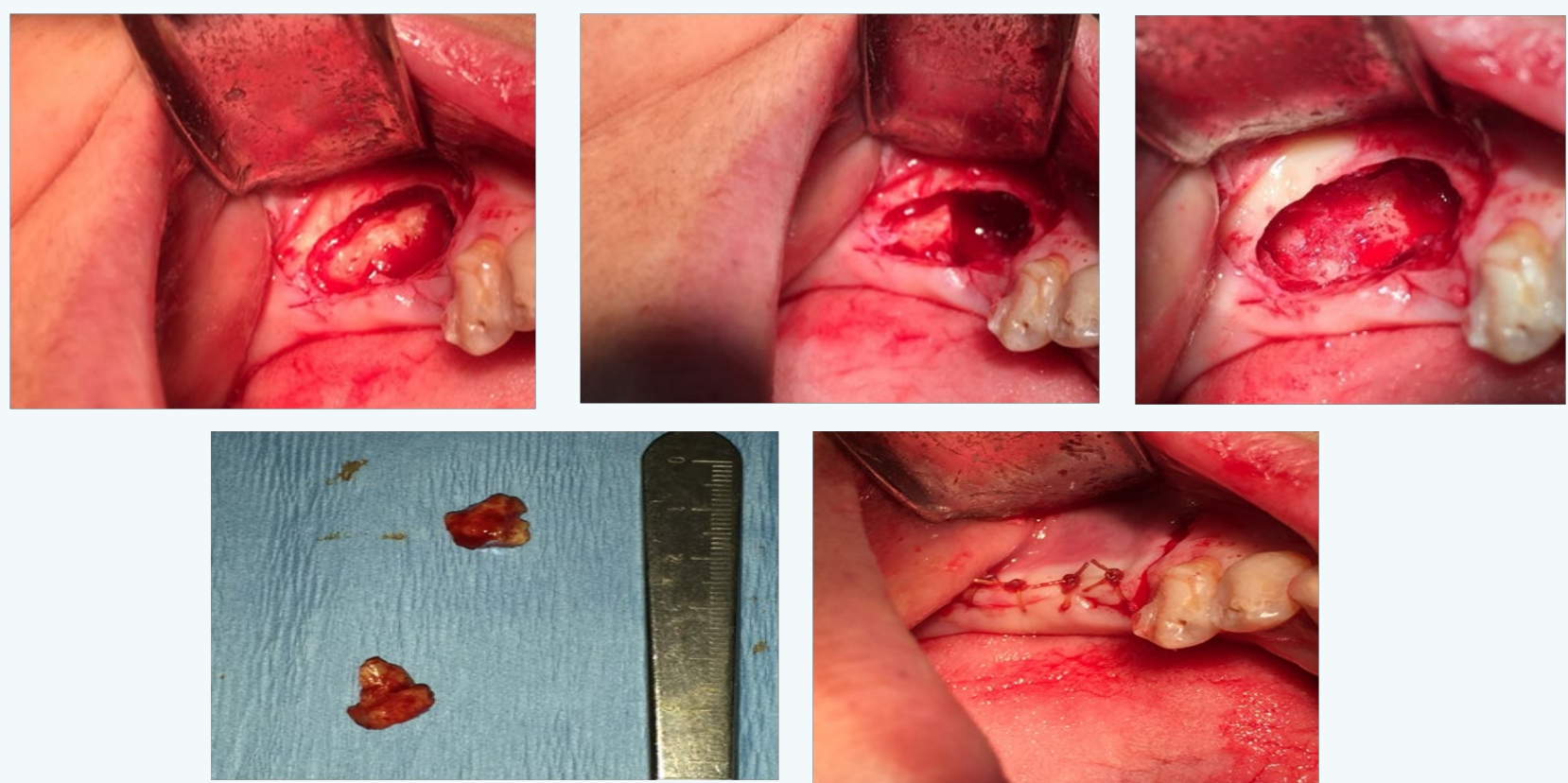

Figures 6-10: The patient presented for surgery. 
The patient presented for surgery (Figures 6-10) and the involucrum was removed under local anesthetic using 108 mg of lidocaine $2 \%$ with epinephrine 1:100.000, the lesion was approached form the most coronal aspect, sectioned in two and removed with a gross description of two fragments of tan-brow hard tissue measuring $2 \times 1 \times 1.5 \mathrm{~cm}$ placed in aggregate), the patient was sent home and a Medrol Dose Pack (methylprednisolone) was prescribe [3-5].

The specimen was submitted for decalcification with a clinical impression of Periapical Osseous Dysplasia, the diagnosis was returned as a Non-Vital Hard Tissue with Abscess, with microscopic description of dense fragments of non-vital cemento -osseous hard tissue with necrotic marrow spaces and associated abscess and some areas of fibro-vascular marrow consistent with an infected benign fibro-osseous lesion (Osseous dysplasia) [6].

Two weeks later the patient presented for removal of sutures and post operative check up, she reported no pain, numbness, bleeding or suppuration. Six months later the patient presented for follow up Cone Beam CT (Figure 11,12), a CBCT scan was taken for post operative evaluation, a limited Volume maxillofacial noncontrast, thin slices $(0.133 \mu \mathrm{m}$ resolution), Cone-Beam CT, $85 \mathrm{kV}$ $10 \mathrm{~mA}$ for $6 \mathrm{~s},\left(503 \mathrm{mGy} . \mathrm{cm}^{2}\right)$ of the left body of the mandible was taken. The scan revealed the entire area where the lesion was located at was filled with a fibrous healing defect (see arrows on the selected reformatted images). The patient has been followed in our practice has been symptoms free for 6 months and is ready for rehabilitation via removable partial dentures.
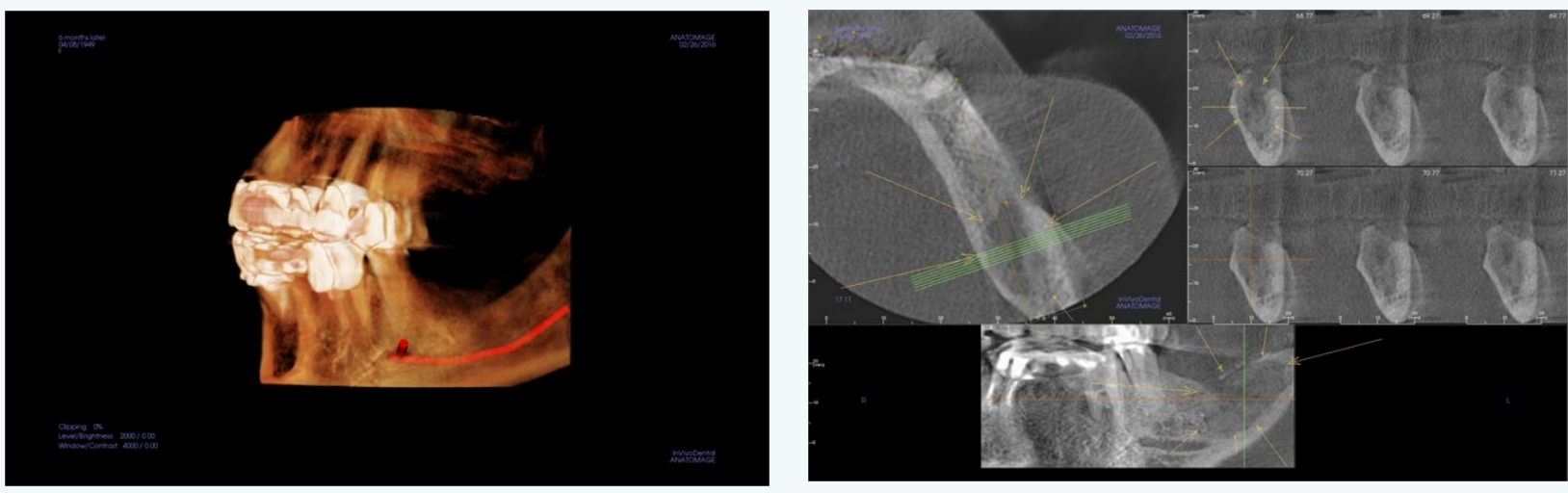

Figures 11 \& 12: Six months later the patient presented for follow up Cone Beam CT.

Note: Multiplanar reformatting was carried out at the work station using InVivo5 Software, with selected images which were adjusted for viewing and are not actual size scale, any measurements or recorded markers have been made in the primary image fields and are included only as guidelines not as substitute for complete analysis using the primary imaging series. Measurements provided are estimates, and may not represent the exact dimensions; actual dimensions will depend on slice selection and orientation. The use of intra-oral radiographs for assessment of caries and periodontal disease was recommended.

\section{References}

1. Mufeed A, Ummar M, Antony George, Abdul Hafiz (2015) Infected florid osseous dysplasia: clinical and imaging follow up. BMJ Case Rep.
2. Pindborg JJ, Kramer IRH, Torloni H (1971) Histological typing of odontogenic tumors, jaws cysts and allied lesions. World Health Organization, Geneva, Switzerland.

3. Melrose RJ, Abrams AM, Mills BG (1976) Florid osseous dysplasia: a clinico-pathologic study of 34 cases. Oral Surg Oral Med Oral Path 41(1): 62-82.

4. Loh FunChee, Yeo Jinn-fei (1989) florid osseous dysplasia in Orientals. Oral Surg Oral Med Oral Path 68(6): 748-753.

5. Stuart C White, Michael Pharoah (2009) Oral Radiology: Principles and interpretation. Mosby $\left(6^{\text {th }}\right.$ edn).

6. Sanjai K, Kumarswamy J, Kumar VK, Archana Patil (2010) Florid cemento osseous dysplasia in association with dentigerous cyst. J Oral Maxillofac Pathol 14(2): 63-68. 